\title{
Guidelines and Safety Practices for Improving Patient Safety
}

\author{
Walter Ricciardi and Fidelia Cascini
}

\subsection{Introduction}

Actions to improve patient safety have shown widely varying degrees of effectiveness. Usually hospitals are focused on the occurrence of adverse events and the level of adversity to the patient in the contexts of insurance premiums and the costs of malpractice. Furthermore, even risk management units within hospitals focus on these factors, when comparing the performance of departments or wards. However, for the improvement of patient safety in clinical practice, a different approach is required, in which the prevention of patient harm and effectiveness of clinical actions is standardized and assessed on the basis of scientific evidence.

Recommendations that have been translated into guidelines are the best possible evidencebased solutions to clinical practice issues. However, it appears that there are very few clinical guidelines focused on patient safety, particularly in the risk management sector. Furthermore, when using clinical guidelines for quality and safety improvement, practices often seem to diverge. Higher quality and safer clinical practice are consequently difficult to achieve, share, and promote.

W. Ricciardi · F. Cascini $(\bowtie)$

Section of Hygiene and Public Health, Università

Cattolica del Sacro Cuore, Rome, Italy

e-mail: walter.ricciardi@unicatt.it;

fidelia.cascini1@unicatt.it
Existing knowledge of patient safety essentially covers the nosography of threats and causes of patient harm, as opposed to possible evidencebased solutions that can (a) prevent risks, (b) address healthcare incidents, and (c) which can be compared. This means that etiology, pathogenesis, and observations of safety issues in clinical departments, and, more broadly in healthcare organizations, are often investigated while proven solutions to patient safety issues are rarely discussed. To give an appropriate analogy, it is like saying that there are many papers that have examined perioperative complications, type of surgeries, and patient characteristics. However, no research is available on how the occurrence of these complications have been managed in different settings according to organizational and human factors.

It is essential that healthcare professionals acquire proficiency in producing evidence that can be used for making improvements to patient's safety and managing the risks of adverse events. To successfully achieve this goal, the first step is for them to have a clear idea of what guidelines and practices are. Definitions of these terms will be the content of the first section of this chapter. Once these concepts have been introduced, the second section will show the current picture regarding patient safety and why a greater number of valuable clinical guidelines are needed. The third section will then consider possible solutions, lessons to apply in practice, and will 
explain how to prepare and update a guideline. The challenges we are facing along with the limits of the current guidelines will be considered at the end, which will assist in managing patient safety in future.

\subsection{The Need to Understand Guidelines Before Improving Safety}

The World Health Organization (WHO) regards guidelines as tools to help people to make decisions and particularly emphasize the concept of choosing from a range of interventions or measures. A WHO guideline is any document developed by the World Health Organization containing recommendations for clinical practice or public health policy. A recommendation tells the intended end-user of the guideline what he or she can or should do in specific situations to achieve the best health outcomes possible, individually or collectively. It offers a choice of different interventions or measures that are intended to have a positive impact on health and explains their implications for the use of resources. Recommendations help the user of the guideline make informed decisions on whether to undertake specific interventions or clinical tests, or if they should implement wider public health measures, as well as where and when to do so. Recommendations also help the user to select and prioritize across a range of potential interventions [1].

With a greater emphasis on clinical practice, the U.S. Institute of Medicine (IOM) defines guidelines as "statements that include recommendations, intended to optimize patient care, that are informed by a systematic review of evidence and an assessment of the benefits and harms of alternative care options" [2]. This definition emphasizes that the foundation of a guideline is a systematic review of the scientific evidence bearing on a clinical issue. The strength of the evidence leads the clinical decision-making process through a set of recommendations. These concern the benefits and harms of alternative care options and address how patients should be managed, everything else being equal.
The U.S. National Guideline Clearinghouse (NGC) of the Agency for Healthcare Research and Quality (AHRQ) also uses the definition of clinical practice guidelines developed by the IOM, stating that "clinical practice guidelines are statements that include recommendations intended to optimize patient care that are informed by a systematic review of evidence and an assessment of the benefits and harms of alternative care options" [3].

The British National Institute for Health and Care Excellence (NICE) stresses scientific evidence as the basis of guidelines. It states: "NICE guidelines make evidence-based recommendations on a wide range of topics, from preventing and managing specific conditions, improving health, and managing medicines in different settings, to providing social care and support to adults and children, safe staffing, and planning broader services and interventions to improve the health of communities" [4].

The Italian National Center for Clinical Excellence (CNEC) that is responsible for the National Guidelines System (SNLG) uses essentially the same definition as NICE, stressing the importance of evidence-based medicine as the foundation of recommendations in guidelines.

The recent report on healthcare quality improvement published by the European Observatory on Health Systems and Policies [5] reiterates that clinical guidelines focus on how to approach patients with defined healthcare problems, either throughout the entire care process or in specific clinical situations. As such, they can be considered as a tool to inform healthcare delivery, with a specific focus on the clinical components, in the context of medical practice as an applied science. Clinical guidelines have the potential to reduce unwarranted practice variation and enhance translation of research into practice; a well-developed guideline which is also well implemented will help improve patient outcomes by optimizing the process of care $[6$, 7].

From the perspective of international accreditation societies such as Joint Commission International (JCI), guidelines that help healthcare organizations to improve performance and 
outcomes are part of the foundation of processes aimed at reaching the goal of safe and highquality care [8]. JCI maintains that clinical practice guidelines are truly major and effective tools in the practice of delivering evidence-based medicine to achieve more effective patient outcomes and safer care. These guidelines, which must be used in all JCI accreditation programs, can achieve their maximum potential when they are both well developed and effectively introduced into clinical practice.

All of the definitions mentioned are consistent. Guidelines are not presented as a substitute for the advice of a physician or other knowledgeable healthcare professionals or providers. They are tools describing recommended courses of intervention whose key elements are the best available scientific evidence and actions according to this evidence. The goal is the promotion of health and consequently, the quality and safety of care. However, it is also desirable for professionals to share within the scientific community the results from using clinical practice guidelines in the context of valuable, real-world experience to inform safety interventions. Professionals are expected to share their current practice to help them apply guidelines to real-life situations and also to improve guidelines in the light of that experience.

Ensuring the quality of healthcare services and making improvements to patient safety require that evidence-based recommendations from guidelines, and their application in the form of practical interventions (best practices), always function as synergetic tools. Nevertheless, there is no consensus on what constitutes practicebased evidence (which is what emerges from routine hospital activities) and what metrics can be used to ensure the quality of this evidence. Healthcare interventions that have been shown to produce desirable outcomes and that are suitable for adaptation to other settings can be called "best practices." A best practice is "an intervention that has shown evidence of effectiveness in a particular setting and is likely to be replicable to other situations" [9]. Moreover, a best practice is not a synonym of a good practice or, simply, of a practice: it is an already existing and selected intervention whose effectiveness has already been established. This concept is widely applicable in health care, from patient safety to public health, including the quality of care. In fact, a best practice is based on evidence from up-todate research and it has the added value of incorporating experience acquired in real-life settings.

A best practice provides tangible solutions as the most effective process or method to achieve a specific objective, with results that are shareable. As a consequence, the practice can then become a model. Some organizations are working on creating best practice models, in particular, on selecting techniques or methodologies that have been proven to be reliable in achieving desired results through consolidated and updated experience and research. The British Medical Journal (BMJ), for example, funds a service (available at https://bestpractice.bmj.com/info/) that collects the latest evidence-based information to support professional decisions and brings together regularly updated research evidence and the knowledge of international experts. According to the BMJ, its best practice tool is "a clinical decision support tool that offers a step-by-step approach to help manage patient diagnosis, prognosis, treatment and prevention."

\subsection{The Current Patient Safety Picture and the Demand for Guidelines}

In most healthcare settings worldwide, patient safety data is data on the absence of patient safety. On the last patient safety day (September 17, 2019), WHO announced, "Patient safety is a serious global public health concern. It is estimated that there is a 1 in 3 million risk of dying while travelling by airplane. In comparison, the risk of patient death occurring due to a preventable medical accident, while receiving health care, is estimated to be 1 in 300" [10]. WHO's message is based on facts found in studies and statistics. These inform us that one in every 10 patients is harmed while receiving hospital care (amounting to nearly $50 \%$ of adverse events 
considered preventable) [11]. Further, the occurrence of adverse events due to unsafe care is one of the 10 leading causes of death and disability across the world [12]. The report of the WHO continues with the following findings [13]:

- Four out of every 10 patients are harmed in primary and outpatient (ambulatory) health care, with up to $80 \%$ of the harm considered to have been preventable.

- Patient harm may account for more than $6 \%$ of hospital bed days and more than 7 million admissions.

- The most detrimental errors are related to diagnosis, prescription, and the use of medicines.

Moreover, there are other serious consequences. The WHO report also included the following criticisms concerning the "health status" of patient safety worldwide: the costs from unsafe medication practices or medication errors [14, 15] and from delayed diagnosis [16, 17], the costs of treating the effects of patient harm, the complications from surgery that cause more than 1 million patient deaths every year [18], and the inappropriate or unskilled use of medical radiation leading to health hazards to both patients and staff [19].

Approaches to improve patient safety have already been suggested. Evidence-based care positively affects healthcare practice and patient outcomes. For example, the United States Agency for Healthcare Research and Quality (AHRQ) [20] stated that the chances of a patient receiving safer care when entering a hospital have increased; an estimated 87,000 fewer patients died from hospital-acquired conditions between 2010 and 2014 in the USA. This not only represents a major improvement in patient safety, but also resulted in estimated savings of $\$ 19.8$ billion. The US Agency reminded noted that hard work to reduce undesired outcomes had been performed by everyone from front-line staff to nurses, physicians, and hospital administrators. Further, theoretical financial savings from safety improvement and patient involvement were identified by WHO [13, 21].
Additional measures to implement safety in practices should be mandatory, such as tools that are mainly evidence-based as well as the education of and outreach to providers and patients, and the widespread use of hospital-based electronic health records. Nevertheless, the practical implementation of evidence-based research to treat unsafe situations remains uncertain. One paradigm case is that of the healthcare-related infections where, although a standardized evidence-based approach to patient safety seems accessible and extremely useful in this field (e.g., hand hygiene guidelines) [22], WHO recently reported [10] that the numbers of healthcare-associated infections remain high, affecting up to 10 out of every 100 hospitalized patients, and that a large proportion were preventable [23-25]. A recent systematic review [26] also observed that $35-55 \%$ of healthcare-associated infections were preventable. This suggests that there remains much to be desired in terms of implementation of evidence-based best practices. Further, the levels of reductions in such infections attributable to the implementation of multifaceted, evidence-based interventions are in line with previous estimates [27, 28].

Even in high-income countries where a high level of adherence to current recommendations is expected, and despite the existence of evidencebased strategies, a further reduction in the occurrence of these infections of $30-50 \%$ is achievable [26]. In reality, a large discrepancy is emerging between the intention to effect change by employing standard operating procedures and the accurate implementation of such practices in daily practice [29]. Great potential exists to further decrease hospital-acquired infection rates in a variety of settings. Relevant factors in the success of such programs include the study design, baseline infection rates and type of infection [30]. Other factors such as global aging trends and comorbidity are likely to require additional efforts to reduce the risk of infections while medical innovations may also reduce this risk due to the emergence of less invasive techniques (e.g., minimally invasive surgery or noninvasive ventilation).

Suggestions for how to address safety improvement in health care can be derived from a 
literature review of evaluations of interventions. The negative impact of failing to improve quality and safety in health care is a public health issue [9]. Instead of simply moving onto the next new paradigm, it is worth considering what deficiencies exist in the literature and how these might be rectified [31].

\section{$1.4 \quad$ Implementing the Research on Patient Safety to Improve Clinical Practice}

Evidence-based medicine is the use of the best available evidence to inform decisions about the care of individual patients [32]. This means that clinical care choices undergo rigorous evaluation instead of having their effectiveness presumed on the basis of subjective experience or arguments relating to the etiopathogenesis of diseases. Despite this, it has been noticed [31] that implementation efforts typically proceed on the basis of intuition, anecdotal stories of success, or studies that exhibit little of the methodological sophistication seen in the research that established the intervention's benefit, even after multiple rigorously designed and well-conducted clinical trials have established the benefit of a particular care process.

Systematic reviews of the evidence and clinical practice guidelines that synthesize studies addressing important clinical decisions have been underestimated in clinical practice. A variety of factors have prevented clinicians from acquiring evidence in a reliable and timely fashion. Such evidence would include factors that have been the object of only limited study so far. Other elements of implementing evidence-based medicine that have been glossed over include the following: disagreement with the content of guidelines, which could quickly become out of date or have wide variations in methodological quality; the personal characteristics of providers, for example, their resistance to perceived infringements on physician autonomy; and logistical or financial barriers [33].

It has also been noted [31] that research into quality improvement (including patient safety) and the related literature differ from the other biomedical research in two major respects. First, evaluations of specific interventions often fail to meet basic standards for the conduct and reporting of research. Second, and more fundamentally, the choices of particular interventions lack compelling theories that can predict their success or be applied to specific features during their development. Methodological shortcomings in the quality improvement research literature include basic problems with the design and analysis of the interventions as well as poor reporting of the results.

In light of this, a recent review [34] highlighted that delivering improvements in the quality and safety of health care remains an international challenge. In recent years, quality improvement methods such as plan-do-study-act (PDSA) cycles have been used in an attempt to drive such improvements. This method is widely used in healthcare improvement however there are little overarching evaluations of how it is applied. PDSA cycles can be used to structure the process of change iteratively, either as a standalone method or as part of a range of quality improvement approaches, such as the Model for Improvement (MFI), Total Quality Management, Continuous QI, Lean, Six Sigma or Quality Improvement Collaboratives [35-37].

Despite the increased use of quality improvement methods, the evidence base for their effectiveness is poor and unsubstantiated [31, 38, 39]. PDSA cycles are often a central component of quality improvement initiatives; however, few formal objective evaluations of their effectiveness or application have been carried out [40]. Some PDSA approaches have been demonstrated to result in significant improvements in care and patient outcomes [41] while others have demonstrated no improvements at all [42-44]. Thus, evidence of effective quality improvement interventions remains mixed, with literature concluding that quality improvement interventions are only effective in specific settings and are used as "single-bullet" interventions that cannot deliver consistent improvements. Conversely, effective interventions need to be complex and multifaceted [45-47] and developed iteratively to adapt to 
the local context and respond to unforeseen obstacles and unintended effects [48, 49].

Finding effective quality improvement methods to support iterative development to test and evaluate interventions in clinical care is essential for the delivery of high-quality and high-value care in a financially constrained environment. However, in the field of quality and safety improvement, strategies for implementing evidence-based medicine require an evidence base of their own, unlike in other medical disciplines [50]. Progress in researching quality improvement requires an understanding of the factors driving provider and organizational change. Moreover, possible elements affecting the results of research when implemented in practice, such as organizational factors and human features related to both professionals and patients, have to be considered. Additionally, research into patient safety improvement and its implementation requires looking at the healthcare system as a whole, including professionals, patients, and features of facilities.

Once an intervention to improve safety has been developed, the next step should be a pilot study to confirm that it works or, in other words, a Phase I of clinical studies [51]. The pilot study should start from a study design that includes the formulation of the hypothesis, the method of sampling the population involved in the study, the choice of and correlations between dependent and independent variables, and the analysis and reporting of results. It is important to ensure that the interpretations and explanations of the efficacy and value of interventions adopted to manage specific patient safety issues are shareable.

Researchers and clinicians working on patient safety improvement should take into consideration the following: how to carry out this particular type of research; if it is correct to consider just a sample or the whole population of patients; what techniques to use in data collection and observation processes; and how to describe the data. All of these elements are essential to support the hypothesis of the study, and to give credibility to both the research meth- odology adopted and the conclusions of the trial. This kind of research is needed to produce informative, reliable, and evidence-based conclusions that ultimately lead to, from a wider point of view, a change of perspective. To be precise, the aim is to switch the focus from the statistics on patient injuries, damages, and claims, to data derived from clinical trials. Ultimately, the purpose of collecting this data is to propose actions and solutions to deal with the lack of safety in healthcare organizations, and medical treatments.

Empirically-derived models are needed to inform decisions to select specific implementation strategies, based on clinical features of the quality target, organizational or social context, and relevant attitudes and beliefs of providers and patients. These models thereby contribute to improvements to quality and the value of the services delivered, and so help to reduce dramatic statistics that can overshadow the vision of a safer healthcare system. It must be noted that although the iterative development of change (PDSA cycle) is the most validated model to improve quality and safety, no single quality improvement tool can absolutely be considered the best. Preferences depend on the skills of professionals and the type of setting which means choosing one method over another for an organization can be difficult.

The choice of the model is an important decision as it can involve serious risks and costly consequences for healthcare organizations. The integration and adaptation of different models to healthcare settings is generally preferable to choosing only one model. However, the problem is that no formal criteria for evaluating the application or reporting of PDSA cycles currently exist. It is only in recent years, through SQUIRE guidelines, that frameworks for publication that explicitly describe PDSA applications have been developed [52, 53]. Such frameworks are necessary to support and assess the effective application of PDSA cycles and to increase their legitimacy as a scientific method for improvement. 


\subsection{Working Towards Producing Guidelines That Improve Safety Practices}

As documents that synthesize current evidence on how to most effectively organize and deliver health services for a given condition [54], guidelines inform healthcare decision-making and can serve as a basis for policy, planning, evaluation, and quality improvement. "Working towards producing guidelines that improve safety practices" means developing structured processes to write, update, and apply guidelines. The most important element to take into account is the methodology. Consequently, it is fundamental to have a plan that is divided into different steps and that can be summarized as a checklist. In fact, a checklist for developing guidelines should contain a comprehensive list of topics and items outlining the practical steps to consider. The checklist is intended for use by guideline developers to plan and track the process of guideline development and to help ensure that no key steps are missed. Following the steps outlined in the checklist ensures that key items are covered and increases the likelihood of the guideline achieving higher scores when evaluated with credibility assessment tools. Checklists for developing guidelines can be combined with guideline credibility assessment tools like AGREE $^{1}$ (Appraisal of Guidelines for Research and Evaluation) [55] and other tools that may reflect standards established by the Guidelines International Network ${ }^{2}$ (GIN) [56] or Institute of Medicine (IOM).

One easy-to-use and reliable checklist is the GIN-McMaster Guideline Development

\footnotetext{
${ }^{1}$ The AGREE (Appraisal of Guidelines for Research and Evaluation) Collaboration developed the most commonly used instrument to assess the quality of a guideline. The instrument comprises 23 criteria grouped in six domains (addressed by the AGREE II): scope and purpose; stakeholder involvement; rigor of development; clarity and presentation; applicability; and editorial independence.

${ }^{2}$ The work of the Guidelines International Network (http:// www.g-i-n.net/) promotes the dissemination of guidelinerelated content and provides an exchange platform for guideline developers and users. Further, the GIN provides reporting guidance for guideline-based performance measurement tools.
}

Checklist, which is available on the internet (https://cebgrade.mcmaster.ca/guidecheck.html). It is divided into 18 steps as follows [57].

1. Organization, budget, planning, and training. These involve outlining a detailed plan describing what is feasible, how it will be achieved, and what resources will be required to produce and use the guideline. The plan should define a specific completion date and be expressed in formal, measurable terms.

2. Priority setting. This refers to the identification, balancing, and ranking of priorities by stakeholders. Priority setting ensures that resources and attention are devoted to those general areas where healthcare recommendations will provide the greatest benefit to the population, jurisdiction, or country, e.g., chronic obstructive pulmonary disease, diabetes, cardiovascular disease, cancer, and prevention. A priority-setting approach needs to contribute to future plans while responding to existing, potentially difficult circumstances.

3. Guideline group membership. This defines who is involved and in what capacity, how the members are selected, and in which steps of the development of the guidelines each of them will participate.

4. Establishing guideline group processes. This defines the steps to be followed, how those involved will interact, and how decisions will be made.

5. Identifying target audience and topic selection. This involves defining the potential users or beneficiaries of the guidelines and defining the topics to be covered in the guideline (e.g., the diagnosis of chronic obstructive pulmonary disease).

6. Beneficiary and other stakeholder involvement. This describes how relevant people or groups who are not necessarily members of the panel (e.g., as the beneficiaries or users) will be affected by the guidelines and involved in their development.

7. Conflict of interest considerations. This focuses on defining and managing the potential divergence between an individual's 
interests and his or her professional obligations. These considerations lead to questions about whether actions or decisions are motivated by gain, such as financial, academic advancement, clinical revenue streams, or community standing. Financial, intellectual, or other relationships that may affect an individual's or organization's ability to approach a scientific question with an open mind are included.

8. Question generation. This focuses on defining key questions the recommendations should address using the PICO (patient/ problem, intervention, comparison, outcome) framework, including the detailed population, intervention (including diagnostic tests and strategies), and outcomes that will be relevant in decision-making (e.g., in chronic obstructive pulmonary disease, should test A or treatments B, C, D, or E be used?).

9. Considering the importance of outcomes and interventions, values, preferences, and advantages. This includes integrating how those affected by recommendations should assess the possible consequences into the process of developing guidelines. These considerations can include: (a) patient, caregiver, and healthcare provider knowledge, attitudes, expectations, moral and ethical values, and beliefs; (b) patient goals for life and health; (c) prior experience with the intervention and the condition; (d) symptoms experienced, e.g., breathlessness, pain, dyspnea, weight loss; (e) preferences relating to and importance of desirable and undesirable outcomes; (f) perceived impact of the condition or interventions on quality of life, wellbeing, or satisfaction; (g) interactions between the work of implementing the intervention, the intervention itself, and patient experiences; (h) preferences for alternative courses of action; and finally, (i) preferences relating to communication content and styles, information and involvement in decision-making and care.

10. Deciding what evidence to include and searching for evidence. This focuses on out- lining inclusion and exclusion criteria based on types of evidence (e.g., rigorous research or anecdotes), study designs, characteristics of the population, interventions, and comparators. It also covers deciding how the evidence will be identified and obtained, which should not be limited to evidence about values and preferences, local data and resources.

11. Summarizing evidence and considering additional information. This focuses on presenting evidence in a synthetic format (e.g., tables or brief narratives) to facilitate the development and understanding of recommendations. It also involves identifying and considering additional information relevant to the question under consideration.

12. Judging quality, strength, or certainty of a body of evidence. This consists of assessing the confidence one can place in the evidence obtained by transparently evaluating the research (individual- and group studies) and other evidence applying structured approaches. This may include, but is not limited to, evidence about baseline risk or burden of disease, the importance of outcomes and interventions, values, preferences, benefits and drawbacks, use of resources (e.g., finance), estimates of effects, and accuracy of diagnostic tests.

13. Developing recommendations and determining their strength. Developing recommendations involves the use of a structured analytical framework and a transparent and systematic process to integrate the factors that influence a recommendation. Determining the strength of the recommendations refers to judgments about how confident a guideline panel is that the implementation of a recommendation will exert a greater number of desirable consequences than undesirable ones.

14. Wording of recommendations and of considerations about implementation, feasibility, and equity. This refers to choosing syntax and formulations that facilitate the understanding and implementation of the recommendations, accounting for the views of the guideline panel. 
15. Reporting and peer review. Reporting refers to how a guideline will be made public (e.g., print, online). Peer review refers to how the guideline document will be reviewed before its publication and how it can be assessed (e.g., for errors), both internally and externally, by stakeholders who were not members of the guideline development group.

16. Dissemination and implementation. This focuses on strategies to make relevant groups aware of the guidelines and to enhance their uptake (e.g., publications and tools such as mobile applications).

17. Evaluation and use. This refers to formal and informal strategies that allow the evaluation of (a) the guidelines as a process and product; (b) their use or uptake, or both; and (c) their impact and whether or not they will lead to improvements in patient or population health or other consequences.

18. Updating. This refers to how and when a guideline will require revision because of changes in the evidence or other factors that influence the recommendations.

All the above-mentioned steps are believed to optimize the development and implementation of guidelines. However, two tough questions on guidelines persist, namely [8]:

(a) Is there enough evidence to make recommendations?

(b) How should we apply recommendations to individual patients?

With respect to the evidence to make recommendations, guideline development tools have, since their inception in 2003, increasingly included the GRADE approach [58-60]. The Grading of Recommendations Assessment, Development and Evaluation (GRADE) approach was created by the eponymous working group (www.gradeworkinggroup.org), which is a collaborative project, consisting mainly of methodologists and clinicians. It provides a framework for assessing the quality (or "certainty") of the evidence supporting, inter alia, guideline recommendations and therefore their resulting strength
[61]. Essentially, GRADE classifies recommendations as "strong" when a specific, recommended intervention or management strategy would be chosen, on reasonable grounds, by a majority of patients, clinicians, or policymakers in all care scenarios. In contrast, such recommendations would be classified as "weak" when there is a reasonable range of choices, reflecting the following possible factors: limited evidence quality, uncertain benefit-harm ratios, uncertainty regarding treatment effects, questionable costeffectiveness, or variability in values and preferences [62]. Further, the GRADE evidence-to-decision framework helps guideline developers to structure their process and evaluation of available evidence [59]. Nonetheless, a trade-off between methodological rigor and pragmatism is required $[63,64]$.

Concerning the issue of applying recommendations to individual patients, it has been observed that practices from guidelines vary considerably and translating guidelines into practice can fail to close gaps that have been identified, both in the scope and the follow-up of interventions [65]. Education for professionals and/or patients is a good strategy to ensure the implementation of guidelines. Nonetheless, another substantial influence on the ability to implement guidelines is how their implementation has already been built into the guideline development process. The planning of implementation provides a set of concrete, actionable steps to take during the implementation phase [66, 67]. The central elements of successful implementation approaches appear in: their target-oriented dissemination, education and training, social interaction, decision support systems and routine procedures, thereby tailoring implementation strategies to settings and target groups [68]. To assist guideline developers regarding implementation, a tool with context-specific implementability features for the whole guideline process has been developed [69].

Further, clinicians must balance the risks and benefits of any guideline recommendation for an individual patient and consider that patient's preferences. If the patient does not adhere to care recommendations, health benefits will not be 
maximized or perhaps even realized. Clinical decisions should be based on guideline recommendations, but all decisions must be individualized according to a patient's risk-benefit ratio and incorporate patient preferences through shared decision-making. Clinician leadership in quality improvement efforts and administrative support are key drivers of quality and safety improvement through care-integrated tools and aligned incentives aimed at achieving meaningful guideline implementation.

One of the most prominent developments in the area of guideline implementation in recent years has been the increased utilization of information technologies to facilitate: (a) push mechanisms for guideline adherence, such as decision support components integrated into clinical management software, for example, alerts, reminders, or routine procedures [70]; (b) the use of guidelines at the bedside, available on, for example, mobile guideline apps; (c) the faster and potentially real-time updating of individual guideline recommendations as new evidence emerges, for example, by adding "living guidelines" [71, 72]. Observational data is necessary to describe current health provision and its quality, pinpoint potential patient groups that are adequately covered by guideline recommendations, and identify gaps and issues to be resolved by clinical research. This data is also vital for identifying late onset treatment harms and drug safety issues.

\subsection{The Challenges of Improving Safety and the Current Limits of Guidelines}

Guidelines are expected to be focused on broad and complex topics, on developing standards to guide healthcare organizations, on providing best practice recommendations for patient care, and on informing the clinical decision-making of health professionals. Successfully incorporating all of these factors into features of guidelines is particularly difficult in today's age of complexity and multimorbidity. This is an age which is also characterized by the desire for personalized medicine and the ambition to push the frontiers of modernization, for example, by introducing artificial intelligence into health care. Thus, beyond the methodological quality of the guideline itself, there are many relevant aspects which represent challenges or limits to take into account regarding guidelines and their applicability.

The first challenge is to improve the effectiveness of a guideline-especially regarding how it improves the safety of care-while also focusing on patient-centeredness; this principle consists of (a) properly taking into account the needs and preferences of patients and of their caregivers and (b) supporting professionals in improving their practice. These dimensions are fundamental to the delivery of care and to patient outcomes as well [73-75]. Patient-centeredness constitutes a more recent focus of the discussion around the development and use of guidelines [76]. Guidelines can facilitate patient education, engagement, and shared decision-making, thus assuring that individual patient values are balanced against the desired outcomes, which are embedded in the trials that form the basis of guideline recommendations. Different modalities of patient involvement exist in different contexts. The two most studied ones are (a) patient group representatives, who are sometimes involved in the guideline development process and (b) guideline documents, which are increasingly produced in different formats for practitioners and patients [77-81].

Another challenge is related to the speed with which medical knowledge progresses and the pace of knowledge production at the primary research level. Guideline recommendations are expected to be kept up to date but a relatively recent, comprehensive review of this issue [82], concluded that 1 in 5 recommendations is out of date 3 years after being launched and that longer updating intervals are potentially too long. Additionally, the development and updating of clinical guidelines represents a challenge because of the speed and resources required for producing and especially updating them. Approaches that can result in efficient and potentially real-time updating of guideline recommendations as new evidence emerges have been discussed, particularly in the form of living systematic reviews and living guidelines [71, 83-85]. 
With regard to limitations, there are different aspects to consider. Maybe the most restrictive limitation regards the evaluation of the costs of the guideline development process, compared with the effectiveness of guidelines, once they are implemented. This limitation particularly relates to the use (or under-use) of costeffectiveness analyses as a part of the development process of clinical guidelines and their related challenges or opportunities [86]. A comprehensive cost-effectiveness analysis should cover the costs of the development and of the guideline dissemination/implementation processes, and the change in the effectiveness of health service by putting the guideline into practice. However, data on the costs of guideline development is scarce and, given the vast variability of settings and practices, likely not generalizable [87]. As has been already pointed out [88], only $27 \%$ of 200 studies on guideline implementation strategies (of which only 11 were from Europe) had some data on cost and only 4 (2\%) provided data on development and implementation. Most of the relevant studies only partially accounted for the costs incurred in the process of guideline production. In some contexts, active implementation seemed to require a substantial upfront investment compared to general dissemination practices. Furthermore, the results regarding optimized processes of care and improved patient outcomes were not sufficient to render them costeffective [89, 90].

Another relevant limitation is that the concept of a guideline-based quality indicator framework has so far been inadequately elaborated, despite the fact that performance measurement sustains the relationship between clinical guidelines and healthcare data. More and more guideline groups have developed quality indicators along with sets of recommendations [91]. Usually, these indicators are primarily intended as general performance measures. However, a closer look at measurement results can provide insights into the extent to which practice reflects guideline recommendations. In other words, the indicators inform us on the extent of guideline adherence, and consequently feed into how they are shaped. Moreover, an overview of country-specific prac- tices [5] clearly demonstrates how divergent guideline practices can be, especially when viewed as strategies for the improvement of healthcare quality. The context-specific nature of guidelines persists, despite their adaptability to the practices of different countries. In the past, the quality of clinical guidelines was narrowly defined according to how closely recommendations were linked to scientific and clinical evidence [92]; however more recently, researchers have explicitly addressed the question of whether guidelines should be systematically pilot-tested in care delivery settings before being finalized [93].

Switching the focus to how guidelines are implemented, newer studies have shown mixed results regarding the effect of guidelines on outcomes but established a clear link between implementation modalities and patient outcomes [94-97]. Barriers to the adoption of or adherence to guidelines by clinicians have been discussed in the literature. Substantial gaps were found in the evidence on the effectiveness of implementation interventions, especially regarding clinical outcomes, cost-effectiveness, and contributory contextual issues [98]. Barriers included time constraints, limited staffing resources, clinician skepticism, clinician knowledge of guidelines, and the age of the clinician. The characteristics of guidelines, such as format, resources, and enduser involvement, were identified as facilitating factors, along with stakeholder involvement, leadership support, and organizational culture (including multidisciplinary teams and electronic guidelines systems).

Beyond challenges and limits, there is the issue of editorial independence in clinical guideline development. Implementing guideline recommendations that have been created in irregular conditions is not only ethically questionable but may also endanger quality of care, as the content may not actually reflect best available evidence. To give an example of irregular conditions, an international survey of 29 institutions involved in clinical guideline development found variability in the content and accessibility of conflict of interest policies; some institutions did not have publicly available policies and, of the policies available, several did not clearly report critical 
steps in obtaining, managing, and communicating disclosure of relationships of interest [99]. While financial conflicts of interest seem to have been adequately disclosed in the most rigorously developed guidelines, active management of existing conflicts of interest has lagged behind [100-103]. Beyond measures to address direct financial conflicts of interest, the management of indirect conflicts of interest is also important in guideline development. Such indirect conflicts can include issues related to academic advancement, clinical revenue streams, community standing, and engagement in academic activities that foster an attachment to a specific point of view [104]. Ensuring that guidelines are developed on the basis of robust consensus processes by a multidisciplinary panel can contribute to mitigating the effects of such conflicts [105].

Systematically developed, evidence-based clinical guidelines are in widespread use as a strategy to improve the quality of healthcare services and consequently the safety of care. However, the rigor of their development, their mode of implementation, and the evaluation of their impacts should be improved in many settings to enable their goal of achieving safer healthcare practices. One of the most important knowledge gaps in this respect is the extent to which guidelines affect patient outcomes and how this effect can be enhanced to ensure better care. For that purpose, both quantitatively measured parameters and patient experience should be taken into account. Today, technology and clinical decision support solutions are readily available to help transform research into practice and recommendations. These solutions take clinically approved best practice guidelines and match them with each patient to provide a recommended and customized care pathway for optimal outcomes. They can also be configured to meet the needs of each organization, taking into consideration local needs and practices [8].

\subsection{Recommendations}

1. The improvement of patient safety should be based on evidence-based recommendations included in well-developed guidelines, which should in turn be rigorously implemented in clinical practice as the best safety practice.

2. More scientific research into healthcare quality and safety improvement is needed, the results and effectiveness of which should be shared across the scientific community worldwide.

3. To face the challenges of a changing healthcare sector in today's age of multi-morbidities, tutors, researchers, caregivers, and patients should work together to address the current limits of clinical guidelines.

\section{References}

1. WHO (World Health Organization). WHO handbook for guideline development. 2nd ed. 2014. https:// apps.who.int/medicinedocs/documents/s22083en/ s22083en.pdf. Accessed 30 Sept 2019.

2. IOM (Institute of Medicine). Clinical practice guidelines we can trust. Washington, DC: The National Academies Press; 2011. http://data. care-statement.org/wp-content/uploads/2016/12/ IOMGuidelines-2013-1.pdf. Accessed 30 Sept 2019.

3. AHRQ (Agency for Healthcare Research and Quality). NGC and NQMC inclusion criteria. 2014. https://www.ahrq.gov/gam/summaries/inclusioncriteria/index.html\#ast. Accessed 30 Sept 2019.

4. NICE (National Institute for Health and Care Excellence). Developing NICE guidelines: the manual. 2014. https://www.nice.org.uk/media/default/ about/what-we-do/our-programmes/developingnice-guidelines-the-manual.pdf. Accessed $30 \mathrm{Sept}$ 2019.

5. European Observatory on Health Systems and Policies. Improving healthcare quality in Europe. Characteristics, effectiveness and implementation of different strategies. Health Policy Series $N^{\circ} 53$. 2019.

6. Qaseem A, et al. Guidelines international network: toward international standards for clinical practice guidelines. Ann Intern Med. 2012;156:525-31.

7. Grimshaw JM, et al. Knowledge translation of research findings. BMC Implement Sci. 2012;7:50.

8. JCI (Joint Commission International). Clinical practice guidelines: closing the gap between theory and practice. 2016.

9. Ng E, de Colombani P. Framework for selecting best practices in public health: a systematic literature review. J Public Health Res. 2015;4:577.

10. WHO (World Health Organization). Patient safety and risk management service delivery and safety. Patient safety fact file. 2019. https://www.who.int/ features/factfiles/patient_safety/patient-safety-factfile.pdf?ua=1. Accessed 30 Sept 2019. 
11. de Vries EN, Ramrattan MA, Smorenburg SM, Gouma DJ, Boermeester MA. The incidence and nature of in-hospital adverse events: a systematic review. Qual Saf Health Care. 2008;17(3):21623. https://doi.org/10.1136/qshc.2007.023622. https://www.ncbi.nlm.nih.gov/pubmed/18519629. Accessed 30 Sept 2019.

12. Jha AK. Presentation at the "Patient Safety-A Grand Challenge for Healthcare Professionals and Policymakers Alike" a roundtable at the grand challenges meeting of the Bill \& Melinda Gates Foundation, 18 Oct 2018. https://globalhealth.harvard.edu/qualitypowerpoint. Accessed 30 Sept 2019.

13. Slawomirski L, Auraaen A, Klazinga N. The economics of patient safety in primary and ambulatory care: flying blind. Paris: OECD; 2018. https://doi. org/10.1787/baf425ad-en. Accessed 1 Oct 2019.

14. Aitken M, Gorokhovich L. Advancing the responsible use of medicines: applying levers for change. Parsippany, NJ: IMS Institute for Healthcare Informatics; 2012. http://papers.ssrn.com/sol3/ papers.cfm?abstract_id=2222541. Accessed 1 Oct 2019.

15. WHO (World Health Organization). Global patient safety challenge: medication without harm. 2017. http://apps.who.int/iris/bitstream/10665/255263/1/ WHO-HIS-SDS-2017.6-eng.pdf?ua=1\&ua=1. Accessed 1 Oct 2019.

16. Khoo EM, Lee WK, Sararaks S, Samad AA, Liew $\mathrm{SM}$, Cheong AT, et al. Medical errors in primary care clinics - a cross sectional study. BMC Fam Pract. 2012;13:127. https://doi.org/10.1186/1471-229613-127. Accessed 1 Oct 2019.

17. National Academies of Sciences, Engineering, and Medicine. Improving diagnosis in health care. Washington, DC: The National Academies Press; 2015. https://www.ncbi.nlm.nih.gov/books/ NBK338596/. Accessed 1 Oct 2019.

18. WHO (World Health Organization). WHO guidelines for safe surgery 2009: safe surgery saves lives. 2009. http://apps.who.int/iris/bitstream/ handle/10665/44185/9789241598552_eng. pdf?sequence=1. Accessed 1 Oct 2019.

19. WHO (World Health Organization). Global initiative on radiation safety in healthcare settings. Technical meeting report. 2008. http://www.who.int/ionizing_radiation/about/GI_TM_Report_2008_Dec.pdf. Accessed 1 Oct 2019.

20. AHRQ (Agency for Healthcare Research and Quality). Saving lives and saving money: hospitalacquired conditions update. Washington, DC; 2015.

21. National scorecard on rates of hospital-acquired conditions 2010 to 2015: Interim data from national efforts to make health care safer. In: Quality and patient safety [website]. Rockville, MD: Agency for Healthcare Research and Quality; 2016. https:// www.ahrq.gov/professionals/qualitypatient-safety/ pfp/2015-interim.html. Accessed 1 Oct 2019.

22. WHO (World Health Organization). WHO guidelines on hand hygiene in health care.
$2009 . \quad$ https://apps.who.int/iris/bitstream/handle/10665/44102/9789241597906_eng.pdf;jsessioni $\mathrm{d}=56 \mathrm{CB} 1 \mathrm{D} 55 \mathrm{BF} 9 A D 7 \mathrm{EA} 4 \mathrm{DCDAC} 163190 \mathrm{~A} 671$ ? equence $=1$. Accessed 1 Oct 2019.

23. WHO (World Health Organization). Report on the burden of endemic health care-associated infection worldwide. 2011. http://apps.who.int/iris/bitstream/handle/10665/80135/9789241501507_eng. pdf? sequence $=1$. Accessed 1 Oct 2019.

24. Suetens C, Latour K, Kärki T, Ricchizzi E, Kinross $\mathrm{P}$, Moro ML, et al. Prevalence of healthcare associated infections, estimated incidence and composite antimicrobial resistance index in acute care hospitals and long-term care facilities: results from two European point prevalence surveys, 2016 to 2017. Euro Surveill. 2018;23(46):1800516. https://www. ncbi.nlm.nih.gov/pmc/articles/PMC6247459/. Accessed 1 Oct 2019.

25. WHO (World Health Organization). Fact sheet: antimicrobial resistance. 2018. https://www.who.int/en/ news-room/fact-sheets/detail/antimicrobial-resistance. Accessed 1 Oct 2019.

26. Schreiber PW, Sax H, Wolfensberger A, Clack L, Kuster SP, Swissnoso. The preventable proportion of healthcare-associated infections 2005-2016: systematic review and meta-analysis. Infect Control Hosp Epidemiol. 2018;39(11):1277-95. https://www.ncbi. nlm.nih.gov/pubmed/30234463. Accessed 1 Oct 2019.

27. Haley RW, Culver DH, White JW, et al. The efficacy of infection surveillance and control programs in preventing nosocomial infections in US hospitals. Am J Epidemiol. 1985;121:182-205.

28. Umscheid CA, Mitchell MD, Doshi JA, Agarwal R, Williams K, Brennan PJ. Estimating the proportion of healthcare-associated infections that are reasonably preventable and the related mortality and costs. Infect Control Hosp Epidemiol. 2011;32:101-14.

29. Sax H, Clack L, Touveneau S, et al. Implementation of infection control best practice in intensive care units throughout Europe: a mixed-method evaluation study. Implement Sci. 2013;8:24.

30. Harbarth S, Sax H, Gastmeier P. The preventable proportion of nosocomial infections: an overview of published reports. J Hosp Infect. 2003;54:258-66.

31. Shojania KG, Grimshaw JM. Evidence-based quality improvement: the state of the science. Health Affairs (Millwood). 2005;24(1):138-50.

32. Sackett DL, et al. Evidence based medicine: what it is and what it isn't (Editorial). Br Med J. 1996;312(7023):71-2.

33. Cabana MD, et al. Why don't physicians follow clinical practice guidelines? A framework for improvement. J Am Med Assoc. 1999;282(15):1458-65.

34. Taylor MJ, Mc Nicholas C, Nicolay C, Darzi A, Bell D, Reed JE. Systematic review of the application of the plan-do-study-act method to improve quality in healthcare. BMJ Qual Saf. 2014;23:290-8.

35. Nicolay CR, Purkayastha S, Greenhalgh A, et al. Systematic review of the application of 
quality improvement methodologies from the manufacturing industry to surgical healthcare. Br J Surg. 2012;99:324-35.

36. Boaden R, Harvey J, Moxham C, et al. Quality improvement: theory and practice in healthcare. Coventry: NHS Institute for Innovation and Improvement; 2008.

37. Schouten LMT, Hulscher MEJL, van Everdingen JJE, et al. Evidence for the impact of quality improvement collaboratives: systematic review. BMJ. 2008;336:1491-4.

38. Walshe K. Understanding what works - and whyin quality improvement: the need for theory-driven evaluation. Int J Qual Health Care. 2007;19:57-9.

39. Auerbach AD, Landefeld CS, Shojania KG. The tension between needing to improve care and knowing how to do it. N Engl J Med. 2007;357:608-13.

40. Ting HH, Shojania KG, Montori VM, et al. Quality improvement science and action. Circulation. 2009; 119:1962-74.

41. Pronovost P, Needham D, Berenholtz S, et al. An intervention to decrease catheter-related bloodstream infections in the ICU. $\mathrm{N}$ Engl $\mathrm{J}$ Med. 2006;355:2725-32.

42. Benning A, Ghaleb M, Suokas A, et al. Large scale organisational intervention to improve patient safety in four UK hospitals: mixed method evaluation. BMJ. 2011;342:d195.

43. Landon BE, Wilson IB, McInnes K, et al. Effects of a quality improvement collaborative on the outcome of care of patients with HIV infection: the EQHIV study. Ann Intern Med. 2004;140:887-96.

44. Vos L, Duckers ML, Wagner C, et al. Applying the quality improvement collaborative method to process redesign: a multiple case study. Implement Sci. 2010;5:19.

45. Oxman AD, Thomson MA, Davis DA, et al. No magic bullets: a systematic review of 102 trials of interventions to improve professional practice. CMAJ. 1995;153:1423.

46. Greenhalgh T, Robert G, Macfarlane F, et al. Diffusion of innovations in service organizations: systematic review and recommendations. Milbank Q. 2004;82:581-629.

47. Plsek PE, Wilson T. Complexity science: complexity, leadership, and management in healthcare organisations. BMJ. 2001;323:746.

48. Damschroder LJ, Aron DC, Keith RE, et al. Fostering implementation of health services research findings into practice: a consolidated framework for advancing implementation science. Implement Sci. 2009;4:50.

49. Powell AE, Rushmer RK, Davies HTO. A systematic narrative review of quality improvement models in health care: NHS Quality Improvement Scotland. Report No. 1844045242. 2009.

50. Grol R, Grimshaw J. Evidence-based implementation of evidence-based medicine. Jt Comm J Qual Improv. 1999;25(10):503-13.
51. Hulscheretal ME. Process evaluation on quality improvement interventions. Qual Saf Health Care. 2003;12(1):40-6.

52. Davidoff F, Batalden P, Stevens D, et al. Publication guidelines for quality improvement in health care: evolution of the SQUIRE project. Qual Saf Health Care. 2008;17(Suppl 1):i3-9.

53. Ogrinc G, Mooney S, Estrada C, et al. The SQUIRE (Standards for Quality Improvement Reporting Excellence) guidelines for quality improvement reporting: explanation and elaboration. Qual Saf Health Care. 2008;17(Suppl 1):i13-32.

54. Weisz G, Cambrosio A, Keating P, Knaapen L, Schlich T, Tournay VJ. The emergence of clinical practice guidelines. Milbank Q. 2007;85(4):691-727.

55. Brouwers M, et al. AGREE II: Advancing guideline development, reporting and evaluation in healthcare. Can Med Assoc J. 2010;182:E839-42.

56. Nothacker $M$, et al. Reporting standards for guideline-based performance measures. Implement Sci. 2016;11:6.

57. Schünemann HJ, Wiercioch W, Etxeandia I, Falavigna M, Santesso N, Mustafa R, Ventresca M, Brignardello-Petersen R, Laisaar KT, Kowalski S, Baldeh T, Zhang Y, Raid U, Neumann I, Norris SL, Thornton J, Harbour R, Treweek S, Guyatt G, Alonso-Coello P, Reinap M, Brožek J, Oxman A, Akl EA. Guidelines 2.0: systematic development of a comprehensive checklist for a successful guideline enterprise. CMAJ. 2014;186:E123-42.

58. Guyatt GH, et al. GRADE guidelines: a new series of articles in the Journal of Clinical Epidemiology. J Clin Epidemiol. 2011;64(4):380-2.

59. Neumann I, et al. The GRADE evidence-to-decision framework: a report of its testing and application in 15 international guideline panels. Implement Sci. 2016;11:93.

60. Khodambashi S, Nytrø $\varnothing$. Reviewing clinical guideline development tools: features and characteristics. BMC Med Inform Decis Mak. 2017;17(1):132.

61. GRADE Working Group. Grading quality of evidence and strength of recommendations. BMJ. 2014;328(7454):1490-4.

62. Vandvik PO, et al. Creating clinical practice guidelines we can trust, use, and share: a new era is imminent. Chest. 2013;144(2):381-9.

63. Browman GP, et al. When is good, good enough? Methodological pragmatism for sustainable guideline development. Implement Sci. 2015;10:28.

64. Richter Sundberg L, Garvare R, Nyström ME. Reaching beyond the review of research evidence: a qualitative study of decision making during the development of clinical practice guidelines for disease prevention in healthcare. BMC Health Serv Res. 2017;17(1):344.

65. Gagliardi AR, Alhabib S, Members of Guidelines International Network Implementation Working Group. Trends in guideline implementation: a scoping systematic review. Implement Sci. 2015;10:54. 
66. Gagliardi AR, et al. Developing a checklist for guideline implementation planning: review and synthesis of guideline development and implementation advice. Implement Sci. 2015;10:19.

67. Richter-Sundberg L, et al. Addressing implementation challenges during guideline development-a case study of Swedish national guidelines for methods of preventing disease. BMC Health Serv Res. 2015;15:19.

68. Fischer F, et al. Barriers and strategies in guideline implementation-a scoping review. Healthcare (Basel, Switzerland). 2016;4(3):36.

69. Brouwers MC, et al. The Guideline Implementability Decision Excellence Model (GUIDE-M): a mixed methods approach to create an international resource to advance the practice guideline field. Implement Sci. 2015;10:36.

70. Wright A, et al. Best practices in clinical decision support: the case of preventive care reminders. Appl Clin Inform. 2010;1(3):331-45.

71. Akl EA, et al. Living systematic reviews: 4. Living guideline recommendations. J Clin Epidemiol. 2017;91:47-53.

72. Thomas J, et al. Living systematic reviews: 2 . Combining human and machine effort. J Clin Epidemiol. 2017;91:31-7.

73. Hewitt-Taylor J. Evidence-based practice, clinical guidelines and care protocols. In: Hewitt-Taylor J, editor. Clinical guidelines and care protocols. Chichester: John Wiley \& Sons; 2017. p. 1-16.

74. May C, Montori VM, Mair FS. We need minimally disruptive medicine. BMJ. 2017;339:b2803.

75. Gupta M. Improved health or improved decision making? The ethical goals of EBM. J Eval Clin Pract. 2011;17(5):957-63.

76. van der Weijden $\mathrm{T}$, et al. How can clinical practice guidelines be adapted to facilitate shared decision making? A qualitative key-informant study. BMJ Qual Saf. 2013;22(10):855-63.

77. Elwyn G, et al. Trustworthy guidelines-excellent; customized care tools-even better. BMC Med. 2015;13:199.

78. Fearns N, et al. What do patients and the public know about clinical practice guidelines and what do they want from them? A qualitative study. BMC Health Serv Res. 2016;16:74.

79. Schipper K, et al. Strategies for disseminating recommendations or guidelines to patients: a systematic review. Implement Sci. 2016;11(1):82.

80. Zhang Y, et al. Using patient values and preferences to inform the importance of health outcomes in practice guideline development following the GRADE approach. Health Qual Life Outcomes. 2017;15(1):52.

81. Cronin RM, et al. Adapting medical guidelines to be patient-centered using a patient-driven process for individuals with sickle cell disease and their caregivers. BMC Hematol. 2018;18:12.

82. Martínez García L, et al. The validity of recommendations from clinical guidelines: a survival analysis. Can Med Assoc J. 2014;186(16):1211-9.
83. Elliott JH, et al. Living systematic reviews: an emerging opportunity to narrow the evidence-practice gap. PLoS Med. 2014;11(2):e1001603.

84. Vernooij RW. Guidance for updating clinical practice guidelines: a systematic review of methodological handbooks. Implement Sci. 2014;9:3.

85. Martínez García L, et al. Efficiency of pragmatic search strategies to update clinical guidelines recommendations. BMC Med Res Methodol. 2015;15:57.

86. Drummond M. Clinical guidelines: a NICE way to introduce cost-effectiveness. Considerations? Value Health. 2016;19(5):525-30.

87. Jensen CE, et al. Systematic review of the costeffectiveness of implementing guidelines on low back pain management in primary care: is transferability to other countries possible? BMJ Open. 2016;6(6):e011042.

88. Vale L, et al. Systematic review of economic evaluations and cost analyses of guideline implementation strategies. Eur J Health Econ. 2017;8(2):111-21.

89. Mortimer D, et al. Economic evaluation of active implementation versus guideline dissemination for evidence-based care of acute low-back pain in a general practice setting. PLoS One. 2013;8(10):e75647.

90. Garrison LP. Cost-effectiveness and clinical practice guidelines: have we reached a tipping point? An overview. Value Health. 2016;19(5):512-5.

91. Blozik E, et al. Simultaneous development of guidelines and quality indicators-how do guideline groups act? A worldwide survey. Int $\mathrm{J}$ Health Care Qual Assur. 2012;25(8):712-29.

92. Heffner JE. Does evidence-based medicine help the development of clinical practice guidelines? Chest. 1998;113(3 Suppl):172S-8S.

93. Li H, et al. A new scale for the evaluation of clinical practice guidelines applicability: development and appraisal. Implement Sci. 2018;13(1):61.

94. Roberts ET, et al. Evaluating clinical practice guidelines based on their association with return to work in administrative claims data. Health Serv Res. 2016;51(3):953-80.

95. Cook DA, et al. Practice variation and practice guidelines: attitudes of generalist and specialist physicians, nurse practitioners, and physician assistants. PLoS One. 2018;13(1):e0191943.

96. Kovacs E, et al. Systematic review and meta-analysis of the effectiveness of implementation strategies for non-communicable disease guidelines in primary health care. J Gen Intern Med. 2018;33(7):1142-54.

97. Shanbhag D, et al. Effectiveness of implementation interventions in improving physician adherence to guideline recommendations in heart failure: a systematic review. BMJ Open. 2018;8(3):e017765.

98. Chan WV, et al. ACC/AHA special report: clinical practice guideline implementation strategies: a summary of systematic reviews by the NHLBI implementation science work group: a report of the American College of Cardiology/American Heart Association Task Force on Clinical Practice Guidelines. J Am Coll Cardiol. 2017;69(8):1076-92. 
99. Morciano C, et al. Policies on conflicts of interest in health care guideline development: a cross-sectional analysis. PLoS One. 2016;11(11):e0166485.

100. Napierala H, et al. Management of financial conflicts of interests in clinical practice guidelines in Germany: results from the public database guideline watch. BMC Med Ethics. 2018;19(1):65.

101. Campsall P, et al. Financial relationships between organizations that produce clinical practice guidelines and the biomedical industry: a cross-sectional study. PLoS Med. 2016;13(5):e1002029.

102. Shnier A, et al. Reporting of financial conflicts of interest in clinical practice guidelines: a case study analysis of guidelines from the Canadian Medical Association Infobase. BMC Health Serv Res. 2016;16:383.
103. Moynihan R, et al. Undisclosed financial ties between guideline writers and pharmaceutical companies: a cross-sectional study across 10 disease categories. BMJ Open. 2019;9:e025864.

104. Schünemann HJ, et al. Guidelines international network: principles for disclosure of interests and management of conflicts in guidelines. Ann Intern Med. 2015;163(7):548-53.

105. Ioannidis JPA. Professional societies should abstain from authorship of guidelines and disease definition statements. Circ Cardiovasc Qual Outcomes. 2018;11(10):e004889.

Open Access This chapter is licensed under the terms of the Creative Commons Attribution 4.0 International License (http://creativecommons.org/licenses/by/4.0/), which permits use, sharing, adaptation, distribution and reproduction in any medium or format, as long as you give appropriate credit to the original author(s) and the source, provide a link to the Creative Commons license and indicate if changes were made.

The images or other third party material in this chapter are included in the chapter's Creative Commons license, unless indicated otherwise in a credit line to the material. If material is not included in the chapter's Creative Commons license and your intended use is not permitted by statutory regulation or exceeds the permitted use, you will need to obtain permission directly from the copyright holder. 